ANALELE ŞTIINȚIFICE ALE UNIVERSITĂŢII “AL.I. CUZA” DIN IAŞI (S.N.)

MATEMATICA, Tomul LXI, 2015, f.1

DOI: $10.2478 /$ aicu-2014-0043

\title{
CYCLIC GROUPS OBTAINED AS QUOTIENT HYPERGROUPS
}

\author{
BY \\ S.SH. MOUSAVI ${ }^{*}$, V. LEOREANU-FOTEA and M. JAFARPOUR
}

\begin{abstract}
We introduce a strongly regular equivalence relation $\rho_{\mathcal{A}}^{*}$ on the hypergroup $H$, such that in a particular case the quotient $\frac{H}{\rho_{\mathcal{A}}^{*}}$ is a cyclic group. Then by using the notion of $\rho_{\mathcal{A}}^{*}$-parts, we investigate the transitivity condition of $\rho_{\mathcal{A}}$. Finally, a characterization of the derived hypergroup $D_{c}(H)$ has been considered.
\end{abstract}

Mathematics Subject Classification 2010: 20N20.

Key words: hypergroup, strongly regular relation, complete parts.

\section{Introduction}

Hyperstructure theory was born in 1934 when MARTY [16] introduced hypergroups as a generalization of groups at the 8th congress of Scandinavian Mathematicions. After that, he proved the utility of hypergroups in solving some problems of groups, algebraic functions and rational fractions. Complete enough surveys of the algebraic hyperstructure theory are the books by Corsini [2], Corsini and Leoreanu [3], Vougiouklis [19]. Some relations in groupoids and hyperstructures which have been considered since the beginning are $\mathcal{A}, \mathcal{A}_{n}, \beta, \beta_{n}, \gamma$ and $\gamma_{n}$, see Corsini [1,4], Davvaz and Karimian [5, 6, 12], Freni [8, 9], Koskas [10, 11], Leoreanu [13, 14], Romeo [17], Vougiouklis [18], Zhan [20] and others. Using the relation $\beta$, complete parts are introduced by Koskas [11] and studied by CorsinI, Migliorato and Sureau $[2,3,15]$. \footnotetext{
ter.

${ }^{*}$ This research has been supported financially by Mahani Mathematical Research Cen-
} 
We present now some basic definitions and results about hypergroups (see [3]).

Let $H$ be a non-empty set and $P^{*}(H)$ be the set of all non-empty subsets of $H$. Let $\star$ be a hyperoperation or a join operation on $\mathrm{H}$, that is, $\star$ is a function from $H \times H$ into $P^{*}(H)$. If $(a, b) \in H \times H$, then its image under $\star$ in $P^{*}(H)$ is denoted by $a \star b$ or $a b$. The join operation is extended to subsets of $H$ in a natural way, that is $A \star B=\bigcup\{a b \mid a \in A, b \in B\}$. The notation $a A$ is used for $\{a\} A$ and $A a$ for $A\{a\}$. Generally, the singleton $\{a\}$ is identified with its member $a$. The structure $(H, \star)$ is called a semihypergroup if $a(b c)=(a b) c$ for all $a, b, c \in H$ and is called a hypergroup if it is semi-hypergroup and $a H=H a=H$ for all $a \in H$.

Let $H$ be a hypergroup and $K$ be a non-empty subset that is closed under the hyperoperation in $H$. If $K$ is itself a hypergroup under the hyperoperation in $H$, then $K$ is said to be a subhypergroup of $H$.

Suppose $(H, \star)$ and $\left(H^{\prime}, \star^{\prime}\right)$ are two semi-hypergroups. A function $f$ : $H \rightarrow H^{\prime}$ is called a homomorphism if $f(a \star b) \subseteq f(a) \star^{\prime} f(b)$ for all $a$ and $b$ in $H$. We say $f$ is a good homomorphism if for all $a$ and $b$ in $H$, $f(a \star b)=f(a) \star^{\prime} f(b)$.

If $(H, \star)$ is a hypergroup and $R \subseteq H \times H$ is an equivalence relation, we set

$$
A \stackrel{\bar{R}}{R} B \Leftrightarrow a R b, \quad \forall a \in A, \forall b \in B,
$$

for all pairs $(A, B)$ of non-empty subsets of $H$. The relation $R$ is said to be left (right) strongly regular if $x R y \Rightarrow a \star x \overline{\bar{R}} a \star y$ (resp. $x R y \Rightarrow x \star a \overline{\bar{R}}$ $y \star a)$, for all $(x, y, a) \in H^{3}$. Moreover, $R$ is called strongly regular if it is both left and right strongly regular.

If $(H, \star)$ is a semi-hypergroup (hypergroup) and $R$ is strongly regular, then the quotient $\frac{H}{R}$ is a semigroup (group respectively) under the operation:

$$
R(x) \otimes R(y)=R(z), \quad \forall z \in x \star y .
$$

For all $n>1$ define the relation $\beta_{n}$ on a semi-hypergroup $H$, as follows:

$$
a \beta_{n} b \Leftrightarrow \exists\left(x_{1}, \ldots, x_{n}\right) \in H^{n}:\{a, b\} \subseteq \prod_{i=1}^{n} x_{i}
$$

and $\beta=\bigcup_{i=1}^{n} \beta_{n}$, where $\beta_{1}=\{(x, x) \mid x \in H\}$ is the diagonal relation $H$. If $\beta^{*}$ is the transitive closure of $\beta$ then $\beta^{*}$ is an equivalence relation (see $[2])$. 
If $H$ is a hypergroup and we consider the canonical projection $\pi: H \rightarrow$ $H / \beta^{*}$ then the heart of $H$ is the inverse image through $\pi$ of the identity of the group $H / \beta^{*}$ and it is denoted by $\omega_{H}$. Hence $\omega_{H}=\pi^{-1}\left(e_{H / \beta^{*}}\right)$.

We call the kernel of a homomorphism $f$, the set $K(f)=f^{-1}\left(\omega_{H^{\prime}}\right)$, where $\omega_{H^{\prime}}$ is the heart of $H^{\prime}$.

The following result was shown by FRENI [7]; another proof can be found in $[2]$.

Theorem 1.1. If $H$ is hypergroup then $\beta=\beta^{*}$.

A non-empty subset $A$ of $H$ is called a complete part of $H$ if for all $n \geqslant 2$ and for all $\left(x_{1}, x_{2}, \ldots, x_{n}\right) \in H^{n}$ we have the following implication:

$$
\prod_{i=1}^{n} x_{i} \bigcap A \neq \emptyset \Rightarrow \prod_{i=1}^{n} x_{i} \subseteq A
$$

A subhypergroup $K$ of a hypergroup $(H, \cdot)$ is called

- closed if from $K \cap a K \neq \emptyset$ or $K \cap K a \neq \emptyset$, where $a \in H$, it follows that $a \in K$;

- invertible if from $a \in b K$ it follows that $b \in a K$ and from $a \in K b$ it follows that $b \in K a$, where $a, b \in H$;

- normal if for all $a \in H, a K=K a$.

Remark 1.2. The kernel of every homomorphism is a complete part and a normal subhypergroup.

Proposition 1.3. Let $K$ be a subhypergroup of a hypergroup $H$.

(i) If $K$ is a complete part, then it is invertible;

(ii) If $K$ is a invertible, then it is closed.

In [8], FRENI introduced the relation $\gamma=\bigcup_{n>1} \gamma_{n}$, where $\gamma_{1}$ is the diagonal relation and for every integer $n>1, \gamma_{n}$ is the relation defined as follows:

$$
x \gamma_{n} y \Leftrightarrow \exists\left(z_{1}, \ldots, z_{n}\right) \in H^{n}, \exists \sigma \in S_{n},: x \in \prod_{i=1}^{n} z_{i}, y \in \prod_{i=1}^{n} z_{\sigma(i)},
$$

where $S_{n}$ is the symmetric group of order $n$. 
In [5], DaVvaz and Karimian introduced the concept of $\gamma$-parts and studied some properties of $C_{\gamma}(A)$, where $C_{\gamma}(A)$ is the intersection of all $\gamma$ parts of a semi-hypergroup $H$. The transitive closure $\gamma^{*}$ of $\gamma$ is the smalest strongly regular equivalence such that the quotient $H / \gamma^{*}$ is commutative semigroup. Moreover, if $H$ is hypergroup then $\gamma$ is transitive (see [8]).

Throughout this paper, $H$ is a hypergroup and $\mathcal{C}=\bigcup_{a \in \mathcal{A}} H_{a}$, where $\emptyset \neq \mathcal{A} \subseteq H, H_{a}=\bigcup a^{n}, n \in \mathbb{N}$ and for all $a, b \in \mathcal{A}$ if $a \neq b$, then $H_{a} \cap H_{b}=\emptyset$.

\section{The relation $\rho_{\mathcal{A}}$}

In this section we introduce the relation $\rho_{\mathcal{A}}$ on a hypergroup $H$, which we use in order to obtain a cyclic group as a quotient structure of $H$. For all $n \geqslant 1$ define $\Re_{n}^{\mathcal{A}}$ as follows $\Re_{n}^{\mathcal{A}}: \stackrel{\text { def }}{=} \Im_{n}^{\mathcal{A}} \cup \wp_{n}^{\mathcal{A}} \cup \jmath_{n}^{\mathcal{A}}$, where

$$
\begin{aligned}
& \Im_{n}^{\mathcal{A}}: \stackrel{\text { def }}{=}\left\{\left(\prod_{i=1}^{n} x_{i}, \prod_{i=1}^{n} y_{i}\right) \mid \exists \sigma \in S_{n}, \prod_{i=1}^{n} y_{i}=\prod_{i=1}^{n} x_{\sigma(i)}\right\}, \\
& \wp_{n}^{\mathcal{A}}: \stackrel{\text { def }}{=}\left\{\left(\prod_{i=1}^{n} x_{i}, \prod_{i=1}^{n} y_{i}\right) \mid \exists a \in \mathcal{A},\left\{x_{1}, \ldots, x_{n}\right\} \cap H_{a}=\left\{y_{1}, \ldots, y_{n}\right\} \cap H_{a} \neq \emptyset,\right. \\
& \left.\exists \sigma_{a} \in S_{n}, \quad y_{j} \in\left\{y_{1}, \ldots, y_{n}\right\} \cap H_{a} \Rightarrow y_{j}=x_{\sigma_{a}(j)}\right\}
\end{aligned}
$$

and

$$
\mathcal{J}_{n}^{\mathcal{A}}: \stackrel{\text { def }}{=}\left\{\left(\prod_{i=1}^{n} x_{i}, \prod_{i=1}^{n} y_{i}\right) \mid\left\{x_{1}, \ldots, x_{n}\right\} \cap \mathcal{C}=\left\{y_{1}, \ldots, y_{n}\right\} \cap \mathcal{C}=\emptyset\right\}
$$

Notice that $\Re_{1}^{\mathcal{A}}: \stackrel{\text { def }}{=}\{(\{x\},\{y\}) \mid\{x, y\} \cap \mathcal{C}=\emptyset$ or $x=y\}$ and

$$
\Re_{2}^{\mathcal{A}}: \stackrel{\text { def }}{=}\left\{(x z, y z),(x z, z y),(z x, z y),(z x, y z) \mid(\{x\},\{y\}) \in \Re_{1}^{\mathcal{A}}, z \in H\right\} .
$$

For every $n \geqslant 1$, define the relation $\rho_{\mathcal{A}}^{n}$ on $H$ by $x \rho_{\mathcal{A}}^{n} y \Leftrightarrow \exists(A, B) \in \Re_{n}^{\mathcal{A}}, x \in$ $A, y \in B$. Notice that for $n=1$ we obtain $x \rho_{\mathcal{A}}^{1} y \Leftrightarrow(\{x\},\{y\}) \in \Re_{1}^{\mathcal{A}}$ or $x=y \in \mathcal{C}$. Obviously, for every $n \geqslant 1$, the relations $\rho_{\mathcal{A}}^{n}$ are symmetric, and the relation $\rho_{\mathcal{A}}=\bigcup_{n \geqslant 1} \rho_{\mathcal{A}}^{n}$ is reflexive and symmetric. Let $\rho_{\mathcal{A}}^{*}$ be the transitive closure of $\rho_{\mathcal{A}}$.

Theorem 2.1. The relation $\rho_{\mathcal{A}}^{*}$ is a strongly regular relation. 
Proof. Notice that $\rho_{\mathcal{A}}^{*}$ is an equivalence relation. In order to prove that it is strongly regular, we have to show first that:

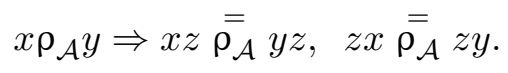

for every $z \in H$. Suppose that $x \rho_{\mathcal{A}} y$, thus there exists $n \geqslant 1$ such that $x \rho_{\mathcal{A}}^{n} y$. Therefore there exists $(B, C) \in \Re_{n}^{\mathcal{A}}$ such that $x \in B$ and $y \in C$. We obtain the following three cases:

Case 1. Suppose that $(B, C) \in \Im_{n}^{\mathcal{A}}$ so we have $B=\prod_{i=1}^{n} x_{i}, C=$ $\prod_{i=1}^{n} y_{i}$ and there exist $\sigma \in S_{n}$ such that $\prod_{i=1}^{n} y_{i}=\prod_{i=1}^{n} x_{\sigma(i)}$. Set $z=$ $y_{n+1}=x_{n+1}$ and let $\tau$ be the permutation of $S_{n+1}$ such that:

$$
\tau(i)= \begin{cases}\sigma(i), & \text { if } i \in\{1,2, \ldots, n\} \\ n+1, & \text { if } i=n+1 .\end{cases}
$$

So $C z=\prod_{i=1}^{n+1} x_{\tau(i)}$ and hence $(B z, C z) \in \Im_{n+1}^{\mathcal{A}}$.

Case 2. Suppose that $(B, C) \in \wp_{n}^{\mathcal{A}}$ so we have $B=\prod_{i=1}^{n} x_{i}, C=$ $\prod_{i=1}^{n} y_{i}$ and there exists $a \in \mathcal{A}$ such that $\left\{x_{1}, \ldots, x_{n}\right\} \cap H_{a}=\left\{y_{1}, \ldots, y_{n}\right\} \cap$ $H_{a} \neq \emptyset$ and $\sigma_{a} \in S_{n}$ such that for all $1 \leqslant j \leqslant n$ if $y_{j} \in H_{a}$, then $y_{j}=x_{\sigma_{a}(j)}$. If $z \notin \mathcal{C}$, then it is easy to see that $(B z, C z) \in \wp_{n+1}^{\mathcal{A}}$. If $z \in \mathcal{C}$, then there exists $b \in \mathcal{A}$ such that $z \in H_{b}$. Set $z=y_{n+1}=x_{n+1}$ and let $\tau_{a}$ be the following permutation of $S_{n+1}$ :

$$
\tau_{a}(i)= \begin{cases}\sigma_{a}(i), & \text { if } i \in\{1,2, \ldots, n\} \\ n+1, & \text { if } i=n+1 .\end{cases}
$$

So for $1 \leqslant j \leqslant n+1$ if $y_{j} \in H_{a}$, then $y_{j}=x_{\tau_{a}(j)}$. Therefore $(B z, C z) \in \wp_{n+1}^{\mathcal{A}}$ and hence $(B z, C z) \in \Re_{n+1}^{\mathcal{A}}$.

Case 3. Suppose that $(B, C) \in \mathcal{J}_{n}^{\mathcal{A}}$ so we have $B=\prod_{i=1}^{n} x_{i}, C=$ $\prod_{i=1}^{n} y_{i},\left\{x_{1}, \ldots, x_{n}\right\} \cap \mathcal{C}=\left\{y_{1}, \ldots, y_{n}\right\} \cap \mathcal{C}=\emptyset$. If $z \notin \mathcal{C}$, then $(B z, C z) \in j_{n+1}^{\mathcal{A}}$ and if $z \in \mathcal{C}$, then $(B z, C z) \in \wp_{n+1}^{\mathcal{A}}$.

Therefore for all $v \in x z$ and for all $y \in y z$, we have $v \in x z \subseteq B z$ and $u \in y z \subseteq C z$, so $v \rho_{\mathcal{A}}^{m} u$ for some $m \in \mathbb{N}$, because $(B z, C z) \in \Re_{m}^{\mathcal{A}}$ for some $m \in \mathbb{N}$ and hence $v \rho_{\mathcal{A}} u$. Thus $x z \bar{\rho}_{\mathcal{A}} y z$. In the same way we obtain that $x \rho_{\mathcal{A}} y \Rightarrow z x \bar{\rho}_{\mathcal{A}} z y$. Moreover, if $x \rho_{\mathcal{A}}^{*} y$, then there exists $m \in \mathbb{N}$ and $\left(u_{0}=x, u_{1}, \ldots, u_{m}=y\right) \in H^{m}$ such that $x=u_{0} \rho_{\mathcal{A}} u_{1} \rho_{\mathcal{A}} \ldots \rho_{\mathcal{A}} u_{m-1} \rho_{\mathcal{A}} u_{m}=$ $y$, whence, by (2.1), we obtain that

$$
x z=u_{0} z \bar{\rho}_{\mathcal{A}} u_{1} z \bar{\rho}_{\mathcal{A}} u_{2} z \bar{\rho}_{\mathcal{A}} \ldots \rho_{\mathcal{A}} u_{m-1} \stackrel{\overline{\rho_{\mathcal{A}}}}{{ }^{\prime}} u_{m} z=y z .
$$


Finally, for all $v \in x z=u_{0} z$ and for all $u \in u_{m} z=y z$, taking $z_{1} \in$ $u_{1} z, z_{2} \in u_{2} z, \ldots, z_{m-1} \in u_{m-1} z$, we have $v \rho_{\mathcal{A}} z_{1} \rho_{\mathcal{A}} z_{2} \rho_{\mathcal{A}} \ldots \rho_{\mathcal{A}} z_{m-1} \rho_{\mathcal{A}} u$, and so $v \rho_{\mathcal{A}}^{*} u$. Therefore $x \rho_{\mathcal{A}}^{*} y \Rightarrow x z \rho_{\mathcal{A}}^{*} y z$.

Similarly we can prove that $x \rho_{\mathcal{A}}^{*} y \Rightarrow z x \rho_{\mathcal{A}}^{*} z y$, hence $\rho_{\mathcal{A}}^{*}$ is strongly regular.

Theorem 2.2. The quotient $\frac{H}{\rho_{\mathcal{A}}^{*}}$ is an abelian group with the generators $\left\{\rho_{\mathcal{A}}^{*}\left(x_{0}\right), \rho_{\mathcal{A}}^{*}(a) \mid x_{0} \in(H-\mathcal{C}), a \in \mathcal{A}\right\}$.

Proof. By Theorem 2.1, $\rho_{\mathcal{A}}^{*}$ is a strongly regular equivalence, so the quotient $\frac{H}{\rho_{\mathcal{A}}^{*}}$ is a group under the following operation $\rho_{\mathcal{A}}^{*}(x) \otimes \rho_{\mathcal{A}}^{*}(y)=$ $\rho_{\mathcal{A}}^{*}(z), \quad \forall z \in x y$.

Since $\gamma^{*} \subseteq \rho_{\mathcal{A}}^{*}$, we conclude that $\frac{H}{\rho_{\mathcal{A}}^{*}}$ is an abelian group. For all $(x, y) \in$ $(H-\mathcal{C})^{2}$ since $\{x, y\} \cap \mathcal{C}=\emptyset$, we have $(\{x\},\{y\}) \in \Re_{1}^{\mathcal{A}}$ and hence $x \rho_{\mathcal{A}}^{*} y$ so $\rho_{\mathcal{A}}^{*}(x)=\rho_{\mathcal{A}}^{*}(y)$. Now suppose that $\rho_{\mathcal{A}}^{*}(h)$ is given. If $h \in(H-\mathcal{C})$, then $\rho_{\mathcal{A}}^{*}(h)=\rho_{\mathcal{A}}^{*}\left(x_{0}\right)$ and if $h \in \mathcal{C}$, then there exists $a \in \mathcal{A}$ such that $h \in H_{a}$. Therefore $\rho_{\mathcal{A}}^{*}(h)=\left[\rho_{\mathcal{A}}^{*}(a)\right]^{k}$ for some $k \in \mathbb{N}$.

Now suppose that $\mathcal{A}=\{a\}$, so $\mathcal{C}=H_{a}$. Put $\eta_{a}^{n} \stackrel{\text { def }}{=} \rho_{\mathcal{A}}^{n}, \eta_{a}=\bigcup_{n \geqslant 1} \eta_{a}^{n}$ and $\eta_{a}^{*}: \stackrel{\text { def }}{=} \rho_{\mathcal{A}}^{*}$. So we have the following corollary:

Corollary 2.3. The quotient $\frac{H}{\eta_{a}^{*}}$ is a cyclic group.

Proof. By the proof of Theorem 2.2, we conclude that the equivalence classes determined by $\rho_{\mathcal{A}}^{*}$ of all elements of $(H-\mathcal{C})$ coincide and the equivalence class of every element of $\mathcal{C}$ is a power of $\eta_{a}^{*}(a)$.

If $H-\mathcal{C} \neq \emptyset$, and $x_{0} \in H-\mathcal{C}$ then there exists $u \in H-\mathcal{C}$ such that $x_{0} \in a u$ since $H$ is a hypergroup. We obtain $\eta_{a}^{*}\left(x_{0}\right)=\eta_{a}^{*}(a) \eta_{a}^{*}(u)=$ $\eta_{a}^{*}(a) \eta_{a}^{*}\left(x_{0}\right)$ whence $\eta_{a}^{*}(a)=e_{\frac{H}{\eta_{a}^{*}}}$ the identity element of the group $\frac{H}{\eta_{a}^{*}}$. Hence $\frac{H}{\eta_{a}^{*}}=<\eta_{a}^{*}\left(x_{0}\right)>$. Moreover, since $a \in x_{0} v$ for some $v \in H-\mathcal{C}$, we obtain that $\eta_{a}^{*}\left(x_{0}\right)=\left(\eta_{a}^{*}\left(x_{0}\right)\right)^{-1}$ which means that the order of $\eta_{a}^{*}\left(x_{0}\right)$ is two, so in this case $\frac{H}{\eta_{a}^{*}}$ is a cyclic group of order two.

Now if $H-\mathcal{C}=\emptyset$, which means that $H$ is a cyclic hypergroup, then $\frac{H}{\eta_{a}^{*}}=<\eta_{a}^{*}(a)>$.

Therefore $\frac{H}{\eta_{a}^{*}}$ is a cyclic group.

Example 2.4. Let $S_{3}$ be the permutation group of order 3, i.e., $S_{3}=$ $\{(1),(12),(13),(23),(123),(132)\}$ and $a=(123)$ be a cycle of order 3 in $S_{3}$. Then $\frac{S_{3}}{\eta_{a}^{*}} \cong \mathbb{Z}_{2}$. 
Proof. It is easy to see that $\Re_{1}^{\mathcal{A}}=\{(x, y) \mid x, y$ are odd permutations or $x=y\}$ and $\Re_{2}^{\mathcal{A}}=\{(z, t) \mid z, t$ are odd or $z, t$ are even permutations $\}$ and $\Re_{n}^{\mathcal{A}}=\Re_{2}^{\mathcal{A}}=\eta_{a}^{*}=\gamma^{*}$, so $\frac{S_{3}}{\eta_{a}^{*}} \cong \mathbb{Z}_{2}$.

Theorem 2.5. The relation $\eta_{a}^{*}$ is the least strongly regular equivalence such that the quotient $\frac{H}{\eta_{a}^{*}}$ is a cyclic group and the equivalence classes of all elements of $(H-\mathcal{C})$ are equal.

Proof. Let $R$ be a strongly regular equivalence such that $\frac{H}{R}$ is a cyclic group and the equivalence classes of $R$ of all elements of $(H-\mathcal{C})$ are equal. Suppose that $\varphi: H \rightarrow \frac{H}{R}$ is the canonical projection. $\varphi$ is a good homomorphism. We show that $\eta_{a}^{*} \subseteq R$.

Let $n \geqslant 1$ and $x \eta_{a}^{n} y$. So there exists $(B, C) \in \Re_{n}^{\mathcal{A}}$ such that $x \in B$ and $y \in C$. We have three cases:

Case 1. Suppose that $(B, C) \in \Im_{n}^{\mathcal{A}}$ so we have $B=\prod_{i=1}^{n} x_{i}, C=$ $\prod_{i=1}^{n} y_{i}$, and there exist $\sigma \in S_{n}$ such that $\prod_{i=1}^{n} y_{i}=\prod_{i=1}^{n} x_{\sigma(i)}$. Since $\varphi(x)=\otimes_{i=1}^{n} \varphi\left(x_{i}\right)$ and $\varphi(y)=\otimes_{i=1}^{n} \varphi\left(y_{i}\right)=\otimes_{i=1}^{n} \varphi\left(x_{\sigma(i)}\right)$, it follows that $\varphi(x)=\varphi(y)$, because $\frac{H}{R}$ is commutative. Therefore $x R y$.

Case 2. Suppose that $(B, C) \in \wp_{n}^{\mathcal{A}}$ so we have $B=\prod_{i=1}^{n} x_{i}, C=$ $\prod_{i=1}^{n} y_{i},\left\{x_{1}, \ldots, x_{n}\right\} \cap H_{a}=\left\{y_{1}, \ldots, y_{n}\right\} \cap H_{a} \neq \emptyset$ and there exists $\sigma \in S_{n}$ such that for all $1 \leqslant j \leqslant n$ if $y_{j} \in H_{a}$, then $y_{j}=x_{\sigma(j)}$. Renumber of the elements of the sets $\left\{x_{1}, \ldots, x_{n}\right\}$ and $\left\{y_{1}, \ldots, y_{n}\right\}$ such that $\left\{y_{1}, \ldots, y_{m}\right\} \subseteq \mathcal{C}$, where $1 \leqslant m \leqslant n$ and $x_{k}, y_{k} \notin \mathcal{C}$ for all $m+1 \leqslant k \leqslant n$. So we have $\varphi(x)=$ $\left(\otimes_{j=1}^{m} \varphi\left(x_{j}\right)\right) \otimes\left(\otimes_{k=m+1}^{n} \varphi\left(x_{k}\right)\right)$ and $\varphi(y)=\left(\otimes_{j=1}^{m} \varphi\left(x_{\sigma(j)}\right)\right) \otimes\left(\otimes_{k=m+1}^{n} \varphi\left(y_{k}\right)\right)$. For all $m+1 \leqslant t, l \leqslant n, \varphi\left(x_{t}\right)=\varphi\left(y_{l}\right)$ and since $\frac{H}{R}$ is a commutative group, we have $\varphi(x)=\varphi(y)$ and hence $x R y$.

Case 3. Suppose that $(B, C) \in \jmath_{n}^{\mathcal{A}}$ so we have $B=\prod_{i=1}^{n} x_{i}, C=$ $\prod_{i=1}^{n} y_{i},\left\{x_{1}, \ldots, x_{n}\right\} \cap \mathcal{C}=\left\{y_{1}, \ldots, y_{n}\right\} \cap \mathcal{C}=\emptyset$. So for all $1 \leqslant i, j \leqslant n, \varphi\left(x_{i}\right)=$ $\varphi\left(y_{j}\right)$, thus $\varphi(x)=\varphi(y)$ and hence $x R y$.

In all cases we have $x R y$ and hence $x \eta_{a} y \Rightarrow x R y$ whence $x \eta_{a}^{*} y \Rightarrow x R y$ by transitivity of $R$. Therefore $\eta_{a}^{*} \subseteq R$.

\section{Transitivity condition of $\rho_{\mathcal{A}}$}

In this section we introduce the concept of $\rho_{\mathcal{A}}^{*}$-part of a hypergroup $H$ and we determine necessary and sufficient conditions such that the relation $\rho_{\mathcal{A}}$ to be transitive. For this purpose, we will follow arguments similar to those exploited by FRENI in [8] in the study of fundamental relation $\gamma$ in hypergroups. 
Definition 3.1. Let $M$ be a non-empty subset of $H$ and $\prod_{i=1}^{n} x_{i} \cap M \neq$ $\emptyset$. We say that $M$ is a $\rho_{\mathcal{A}}^{*}$-part of $H$ if the following conditions hold:

(P1) For all $\sigma \in S_{n}, \prod_{i=1}^{n} x_{\sigma(i)} \subseteq M$;

(P2) If $\exists a \in \mathcal{A},\left\{x_{1}, \ldots, x_{n}\right\} \cap H_{a} \neq \emptyset$ implies that for all $\prod_{i=1}^{n} y_{i}$ such that $\left\{y_{1}, \ldots, y_{n}\right\} \cap H_{a}=\left\{x_{1}, \ldots, x_{n}\right\} \cap H_{a}$ there exists $\sigma_{a} \in S_{n}$ for which $\forall y_{j} \in\left\{y_{1}, \ldots, y_{n}\right\} \cap H_{a}, y_{j}=x_{\sigma_{a}(j)}$, then $\prod_{i=1}^{n} y_{i} \subseteq M$;

(P3) If $\left\{x_{1}, \ldots, x_{n}\right\} \cap \mathcal{C}=\emptyset$ then for all $\prod_{i=1}^{n} y_{i}$ such that $\left\{y_{1}, \ldots, y_{n}\right\} \cap \mathcal{C}=$ $\emptyset$, we have $\prod_{i=1}^{n} y_{i} \subseteq M$.

Proposition 3.2. Let $M$ be a non-empty subset of a hypergroup $H$. The following conditions are equivalent:

(i) $M$ is a $\rho_{\mathcal{A}}^{*}$-part;

(ii) $x \in M, x \rho_{\mathcal{A}} y \Rightarrow y \in M$;

(iii) $x \in M, x \rho_{\mathcal{A}}^{*} y \Rightarrow y \in M$.

Proof. (i) $\Rightarrow$ (ii) Suppose that the pair $(x, y) \in H^{2}$ is such that $x \in M$ and $x \rho_{\mathcal{A}} y$. Then there exists $(B, C) \in \Re_{n}^{\mathcal{A}}$ such that $x \in B$ and $y \in C$. So we have three cases.

Case 1. Let $(B, C) \in \Im_{n}^{\mathcal{A}}$ so we have $B=\prod_{i=1}^{n} x_{i}, C=\prod_{i=1}^{n} y_{i}$ and there exists $\sigma \in S_{n}$ such that $\prod_{i=1}^{n} y_{i}=\prod_{i=1}^{n} x_{\sigma(i)}$. Since $x \in \prod_{i=1}^{n} x_{i} \cap M$, by $(P 1)$ we have $\prod_{i=1}^{n} x_{\sigma(i)} \subseteq M$ and hence $y \in M$.

Case 2. Let $(B, C) \in \wp_{n}^{\mathcal{A}}$. So $B=\prod_{i=1}^{n} x_{i}, C=\prod_{i=1}^{n} y_{i}$, there exists $a \in \mathcal{A}$, such that $\left\{x_{1}, \ldots, x_{n}\right\} \cap H_{a}=\left\{y_{1}, \ldots, y_{n}\right\} \cap H_{a} \neq \emptyset$ and there exists $\sigma_{a} \in S_{n}$ such that for all $1 \leqslant j \leqslant n$ if $y_{j} \in H_{a}$, then $y_{j}=x_{\sigma_{a}(j)}$. Since $x \in \prod_{i=1}^{n} x_{i} \cap M$, by (P2) we have $\prod_{i=1}^{n} y_{i} \subseteq M$ and hence $y \in M$.

Case 3. Suppose that $(B, C) \in \jmath_{n}^{\mathcal{A}}$ so we have $B=\prod_{i=1}^{n} x_{i}, C=$ $\prod_{i=1}^{n} y_{i},\left\{x_{1}, \ldots, x_{n}\right\} \cap \mathcal{C}=\left\{y_{1}, \ldots, y_{n}\right\} \cap \mathcal{C}=\emptyset$. Since $x \in \prod_{i=1}^{n} x_{i} \cap M$, by (P3) we have $\prod_{i=1}^{n} y_{i} \subseteq M$ and hence $y \in M$.

(ii) $\Rightarrow$ (iii) Suppose that $(x, y) \in H^{2}$ is such that $x \in M$ and $x \rho_{\mathcal{A}}^{*} y$. So there exist $m \in \mathbb{N}$ and $\left(w_{0}=x, w_{1}, \ldots, w_{m-1}, w_{m}=y\right) \in H^{m}$ such that $x=w_{0} \rho_{\mathcal{A}} w_{1} \rho_{\mathcal{A}} w_{2} \ldots \rho_{\mathcal{A}} w_{m-1} \rho_{\mathcal{A}} w_{m}=y$. Since $x \in M$, applying (ii) $m$ times, we obtain $y \in M$.

(iii) $\Rightarrow$ (i) Let $x \in \prod_{i=1}^{n} x_{i} \cap M$. We shall check the conditions (P1), (P2) and (P3).

(P1) Let $\sigma \in S_{n}$ be given, thus $\left(\prod_{i=1}^{n} x_{i}, \prod_{i=1}^{n} x_{\sigma(i)}\right) \in \Im_{n}^{\mathcal{A}}$. So for all $y \in \prod_{i=1}^{n} x_{\sigma(i)}$ we have $x \rho_{\mathcal{A}}^{*} y$ and hence $y \in M$. Therefore $\prod_{i=1}^{n} x_{\sigma(i)} \subseteq M$.

(P2) Let $\left\{x_{1}, \ldots, x_{n}\right\} \cap H_{a} \neq \emptyset$ for some $a \in \mathcal{A}$. Now suppose that for all $\prod_{i=1}^{n} y_{i}$ if $\left\{y_{1}, \ldots, y_{n}\right\} \cap H_{a}=\left\{x_{1}, \ldots, x_{n}\right\} \cap H_{a}$ there exists $\sigma_{a} \in S_{n}$ such that 
$\forall y_{j} \in\left\{y_{1}, \ldots, y_{n}\right\} \cap H_{a}, y_{j}=x_{\sigma_{a}(j)}$. Thus $\left(\prod_{i=1}^{n} x_{i}, \prod_{i=1}^{n} y_{i}\right) \in \wp_{n}^{\mathcal{A}}$ and hence for all $y \in \prod_{i=1}^{n} y_{i}$ we have $x \rho_{\mathcal{A}}^{*} y$. Therefore $y \in M$ and so $\prod_{i=1}^{n^{n}} y_{i} \subseteq M$.

(P3) Let $\left\{x_{1}, \ldots, x_{n}\right\} \cap \mathcal{C}=\emptyset$ and $\prod_{i=1}^{n} y_{i}$ be such that $\left\{y_{1}, \ldots, y_{n}\right\} \cap \mathcal{C}=\emptyset$. Thus $\left(\prod_{i=1}^{n} x_{i}, \prod_{i=1}^{n} y_{i}\right) \in \jmath_{n}^{\mathcal{A}}$ and hence for all $y \in \prod_{i=1}^{n} y_{i}$ we have $x \rho_{\mathcal{A}}^{*} y$. Therefore $y \in M$ and so $\prod_{i=1}^{n} y_{i} \subseteq M$.

Notation 3.3. Let $x$ be an arbitrary element of a hypergroup $H$.

For $n \geqslant 1$, set $P_{n}(x)=P_{\Im_{n}}(x) \cup P_{\jmath_{n}}(x) \cup P_{\wp_{n}}(x)$, where

$$
\begin{aligned}
& P_{\Im_{n}}(x)=\bigcup\left\{\prod_{i=1}^{n} x_{\sigma(i)} \mid x \in \prod_{i=1}^{n} x_{i}\right\} \\
& P_{\wp_{n}}(x)=\bigcup\left\{\prod_{i=1}^{n} y_{i} \mid x \in \prod_{i=1}^{n} x_{i},\left\{y_{1}, \ldots, y_{n}\right\} \cap H_{a}=\left\{x_{1}, \ldots, x_{n}\right\} \cap H_{a}\right. \\
& \left.\exists \sigma_{a} \in S_{n}, y_{j} \in\left\{y_{1}, \ldots, y_{n}\right\} \cap H_{a} \Rightarrow y_{j}=x_{\sigma_{a}(j)}\right\} \\
& P_{\jmath_{n}}(x)=\bigcup\left\{\prod_{i=1}^{n} y_{i} \mid x \in \prod_{i=1}^{n} x_{i},\left\{y_{1}, \ldots, y_{n}\right\} \cap \mathcal{C}=\left\{x_{1}, \ldots, x_{n}\right\} \cap \mathcal{C}=\emptyset\right\} .
\end{aligned}
$$

Denote $P(x)=\bigcup_{n \geqslant 1} P_{n}(x)$.

Notice that if $x \notin \mathcal{C}$, then $P_{1}(x)=H-\mathcal{C}$ and

$$
P_{2}(x)=\bigcup_{z \in H}\{d z, z d \mid d \in(H-\mathcal{C}), \exists b \in(H-\mathcal{C}), x \in b z \cup z b\},
$$

while if $x \in a^{n} \subseteq \mathcal{C}$ for some $n \in \mathbb{N}$, then $P_{m}(x)=a^{n}$ for all $m \geqslant 1$.

We have the following:

Proposition 3.4. For every $x \in H, P(x)=\left\{y \in H \mid x \rho_{\mathcal{A}} y\right\}$.

Proof. Suppose that $x \in H$ and $y \in P(x)$ are given. Then there exist $B$ and $C$ such that $x \in B, y \in C$ and:

(i) $y \in P_{\Im_{n}}(x) \Rightarrow(B, C) \in \Im_{n}^{\mathcal{A}}$;

(ii) $y \in P_{\wp_{n}}(x) \Rightarrow(B, C) \in \wp_{\eta}^{\mathcal{A}}$;

(iii) $y \in P_{\jmath_{n}}(x) \Rightarrow(B, C) \in \jmath_{n}^{\mathcal{A}}$.

Therefore $x \rho_{\mathcal{A}} y$ and so $P(x) \subseteq\left\{y \in H \mid x \rho_{\mathcal{A}} y\right\}$.

The proof of the reverse of the inclusion is similar to the above.

Lemma 3.5. Suppose that $H$ is a hypergroup and $M$ is a $\rho_{\mathcal{A}}^{*}$-part of $H$. If $x \in M$, then $P(x) \subseteq M$. 
Proof. It follows by Definition 3.1.

Theorem 3.6. Let $H$ be a hypergroup. The following conditions are equivalent:

(i) $\rho_{\mathcal{A}}$ is transitive;

(ii) for every $x \in H, \rho_{\mathcal{A}}^{*}(x)=P(x)$;

(iii) for every $x \in H, P(x)$ is a $\rho_{\mathcal{A}}^{*}$-part of $H$.

Proof. (i) $\Rightarrow$ (ii) By Proposition 3.4, for all pair $(x, y) \in H^{2}$ we have: $y \in \rho_{\mathcal{A}}^{*}(x) \Leftrightarrow x \rho_{\mathcal{A}}^{*} y \Leftrightarrow x \rho_{\mathcal{A}} y \Leftrightarrow y \in P(x)$.

(ii) $\Rightarrow$ (iii) By Proposition 3.2, if $M$ is a non-empty subset of $H$, then $M$ is a $\rho_{\mathcal{A}}^{*}$-part of $H$ if and only if it is a union of equivalence classes modulo $\rho_{\mathcal{A}}^{*}$. Particularly, every equivalence class modulo $\rho_{\mathcal{A}}^{*}$ is a $\rho_{\mathcal{A}}^{*}$-part of $H$.

(iii) $\Rightarrow$ (i) Suppose that $x \rho_{\mathcal{A}} y$ and $y \rho_{\mathcal{A}} z$ so $x \in P(y)$ and $y \in P(z)$. By Lemma 3.5, we have $P(y) \subseteq P(z)$ and hence $x \in P(z)$. By Proposition 3.4 it follows that $x \rho_{\mathcal{A}} z$ and the proof is complete.

\section{A characterization of a new derived hypergroup of a hy- pergroup}

Let $H$ be a hypergroup and $A$ be an invertible subhypergroup of $H$. For all $x \in H$, there exists $y \in H$ such that $x \in A y$. Therefore the relation ${ }_{A} E$ on $H$ defined by: $x_{A} E y \Leftrightarrow x \in A y$ is a regular equivalence and a congruence. Moreover the quotient $\frac{H}{A^{E}}=\{A x \mid x \in H\}$ is a hypergroup with respect to the hyperoperation $A x \odot A y=\{A z \mid z \in x A y\}$. and the canonical projection $\pi: H \rightarrow \frac{H}{A E}$ is an almost strong homomorphism (see [2]). This means that if $\pi(x)=\pi(a) \odot \pi(t)$, then there exist $a^{\prime}, t^{\prime} \in H$ such that $\pi(a)=\pi\left(a^{\prime}\right), \pi(t)=\pi\left(t^{\prime}\right)$ and $x \in a^{\prime} t^{\prime}$.

In this section we suppose that $\mathcal{A}=\{a\}$ and $\eta_{a}^{*}(a) \neq e_{\frac{H_{i}^{*}}{\eta_{a}^{*}}}$.

Proposition 4.1. For all $x \in(H-\mathcal{C}), \eta_{a}^{*}(x)=e_{\frac{H}{\eta_{a}^{*}}}$.

Proof. Suppose that $\eta_{a}^{*}(x) \neq e_{\frac{H}{\eta_{a}^{*}}}$. By Theorem 2.5, $\frac{H}{\eta_{a}^{*}}=<\eta_{a}^{*}(a)>$, so $\eta_{a}^{*}(x)=\eta_{a}^{*}(t)$ such that $t \in a^{n}$ for some $n \in \mathbb{N}$. Therefore $x \eta^{*} t$ and hence there exist the elements $x=x_{1}, \ldots, x_{n-2}, x_{n-1}, x_{n}=t$ in $H$ such that $x=x_{1} \eta_{a} \ldots x_{n-2} \eta_{a} x_{n-1} \eta_{a} x_{n}=t$. Thus $x_{n-1} \eta_{a} t$ and by Proposition 3.4 it follows that $x_{n-1} \in P(t)$. Since $t \in a^{n}, P(t)=a^{n}$ and hence $x_{n-1} \in a^{n}$. Similarly from $x_{n-2} \eta_{a} x_{n-1}$ we obtain $x_{n-2} \in a^{n}$. After $(n-1)$ steps, we conclude that $x=x_{1} \in a^{n} \subseteq \mathcal{C}$ and it is a contradiction. 
For all pair $(a, b) \in H^{2}$ and for all pair $(A, B)$ of non-empty subsets of $H$, we set:

$$
\begin{aligned}
& a / b=\{x \in H \mid a \in x b\}, \quad a \backslash b=\{x \in H \mid b \in a x\} \\
& A / B=\bigcup_{a \in A, b \in B} a / b, \quad A \backslash B=\bigcup_{a \in A, b \in B} a \backslash b .
\end{aligned}
$$

Denote

$$
D_{1}=\bigcup_{(x, y) \in H^{2}} x y / y x, D_{2}=\bigcup_{(x, y) \in H^{2}} x y \backslash y x, D_{3}=H-\mathcal{C}
$$

and $D_{c}=D_{1} \cup D_{2} \cup D_{3}$.

Define the derived hypergroup $D_{c}(H)$ as the intersection of all subhypergroups, that are complete parts and contain $D_{c}$. Set $D=D_{1} \cup D_{2}$. Since $D \subseteq D_{c}(H)$, it follows that $\frac{H}{D_{c}(H)} E$ is a commutative group (see [8]).

Proposition 4.2. If $H$ is a hypergroup, then $\eta_{a}^{*} \subseteq D_{c}(H) E$.

Proof. Let $x, y \in(H-\mathcal{C})$, so $x, y \in D_{c}(H)$. Since $H$ is a hypergroup, there exists $d \in H$ such that $x \in d y$. Moreover $D_{c}(H)$ is a complete part, so it is closed by Proposition 1.3 and hence $d \in D_{c}(H)$. Therefore $x_{D_{c}(H)} E y$ and so $x$ and $y$ have the same equivalence class under the relation $D_{c}(H) E$.

Now suppose that $x \in a^{n} \subseteq \mathcal{C}$. If $n=1$, then $x_{D_{c}(H)} E a$. Let $n>1$, we have $\pi(x)=\pi(a)^{n}$, where $\pi: H \rightarrow \frac{H}{D_{c}(H)} E$ is the canonical projection. Thus $\pi(x)=\pi(a) \odot \pi(t)$, where $t \in a^{n-1}$. Since $\pi$ is almost strong, there exist $a^{\prime}, t^{\prime} \in H$ such that $\pi(a)=\pi\left(a^{\prime}\right), \pi(t)=\pi\left(t^{\prime}\right)$ and $x \in a^{\prime} t^{\prime}$, so $a^{\prime} t^{\prime} \subseteq D_{c}(H) a D_{c}(H) t$. Since $D_{c}(H)$ is the kernel of $\pi, D_{c}(H)$ is normal and hence $a^{\prime} t^{\prime} \subseteq D_{c}(H) a t$. From $a t \subseteq a^{n}$, there exists $s \in a^{n}$ such that $x \in D_{c}(H) s$. Therefore ${ }_{D_{c}(H)} E(x)={ }_{D_{c}(H)} E(s)$, for some $s \in a^{n}$.

Hence $\frac{H}{D_{c}(H) E}$ is a cyclic group. By Theorem 2.5 it follows that $\eta_{a}^{*} \subseteq D_{D_{c}(H)} E$.

Theorem 4.3. Let $H$ be a hypergroup. If $\phi_{c}$ is the canonical projection $H \stackrel{\phi_{c}}{\longrightarrow} \frac{H}{\eta_{a}^{*}}$, then $D_{c}(H)=\phi_{c}^{-1}\left(e_{\frac{H}{\eta_{a}^{*}}}\right)$.

Proof. Let $z \in D_{c}$, so $z \in D$ or $z \in D_{3}$. If $z \in D$, then $\eta_{a}^{*}(z)=e_{\frac{H}{\eta_{a}^{*}}}$ (see [8]). If $z \in D_{3}$, then $z \in(H-\mathcal{C})$. By Proposition 4.1 we have $\eta_{a}^{*}(z) \stackrel{\frac{H}{\eta_{a}}=}{=}$ 
$e_{\frac{H}{\eta_{a}^{*}}}$. Therefore $D_{c} \subseteq \phi_{c}^{-1}\left(e_{\frac{H}{\eta_{a}^{*}}}\right)$. Since $\eta_{a}^{*}$ is strongly regular, $\phi_{c}^{-1}\left(e_{\frac{H}{\eta_{a}^{*}}}\right)$ is a complete part subhypergroup of $H$, whence $D_{c}(H) \subseteq \phi_{c}^{-1}\left(e_{\frac{H}{\eta_{a}^{*}}}\right)$.

Conversely, for all $x \in \phi_{c}^{-1}\left(e_{\frac{H}{\eta_{a}^{*}}}\right)$ we have $\phi_{c}(\varepsilon)=e_{\frac{H}{\eta_{a}^{*}}}=\phi_{c}(x)$, where $\varepsilon \in D_{c}(H)$. So we obtain $\eta_{a}^{*}(x)=\eta_{a}^{*}(\varepsilon)$ and hence $x \eta_{a}^{*} \varepsilon$. From Proposition 4.2 it follows that $x{ }_{D_{c}(H)} E \varepsilon$ and hence $x \in D_{c}(H) \varepsilon$. Since $D_{c}(H)$ is complete part, by Proposition 1.3 we have $D_{c}(H) x=D_{c}(H)$ whence $x \in D_{c}(H)$. Thus $\phi_{c}^{-1}\left(e_{\frac{H}{\eta_{a}^{*}}}\right) \subseteq D_{c}(H)$ and the proof is complete.

Proposition 4.4. If $M$ is a non-empty subset of a hypergroup $H$, then (i) $\phi_{c}^{-1}\left(\phi_{c}(M)\right)=D_{c}(H) M=M D_{c}(H)$;

(ii) $M$ is an $\eta_{a}^{*}$-part if and only if $\phi_{c}^{-1}\left(\phi_{c}(M)\right)=M$.

Proof. (i) For every $x \in D_{c}(H) M$, there exists a pair $(d, m) \in D_{c}(H) \times$ $M$ such that $x \in d m$ and so $\phi_{c}(x)=\phi_{c}(d) \otimes \phi_{(}(m)=e_{\frac{H_{n}^{*}}{\eta_{a}^{*}}} \otimes \phi_{c}(m)$ by Theorem 4.3. So $\phi_{c}(x)=\phi_{c}(m) \in \phi_{c}(M)$ and hence $x \in \phi_{c}^{-1}\left(\phi_{c}(M)\right)$. Conversely, for all $x \in \phi_{c}^{-1}\left(\phi_{c}(M)\right)$, an element $m \in M$ exists such that $\phi_{c}(x)=\phi_{c}(m)$. Moreover, there exists $d \in H$ such that $x \in d m$, so $\phi_{c}(x)=$ $\phi_{c}(m)=\phi_{c}(d) \otimes \phi_{c}(m)$, whence $\phi_{c}(d)=e_{\frac{H}{\eta_{a}^{*}}}$, and $d \in \phi_{c}^{-1}\left(e_{\frac{H}{\eta_{a}^{*}}}\right)=D_{c}(H)$. Therefore $x \in D_{c}(H) M$.

(ii) Let $M$ be an $\eta_{a}^{*}$-part of $H$ and set $x \in \phi_{c}^{-1}\left(\phi_{c}(M)\right)$. Then there exists $m \in M$ such that $\phi_{c}(x)=\phi_{c}(m)$. So $m \eta_{a}^{*} x$ and by Proposition 3.2 from $m \in M$ it follows that $x \in M$ and hence $\phi_{c}^{-1}\left(\phi_{c}(M)\right) \subseteq M$. It is obvious that $M \subseteq \phi_{c}^{-1}\left(\phi_{c}(M)\right)$ and so the proof is complete.

Theorem 4.5. If $H$ is a hypergroup, then $\eta_{a}$ is transitive.

Proof. By Theorem 3.6, it is enough to show that for all $x \in H, P(x)$ is an $\eta_{a}^{*}$-part of $H$. We check that $\phi_{c}^{-1}\left(\phi_{c}(P(x))\right)=P(x)$ and then we apply Proposition 4.4.

Set $z \in \phi_{c}^{-1}\left(\phi_{c}(P(x))\right)$, so there exists $k \in P(x)$ such that $\phi_{c}(z)=\phi_{c}(k)$ and hence $\eta_{a}^{*}(z)=\eta_{a}^{*}(k)$. By Proposition 3.4 from $k \in P(x)$ it follows that $x \eta_{a} k$. Thus $\eta_{a}^{*}(k)=\eta_{a}^{*}(x)$ and so $\eta_{a}^{*}(z)=\eta_{a}^{*}(x)$. Therefore there exist $z=x_{1}, x_{2}, \ldots, x_{n-1}, x_{n}=x$ such that:

$$
z=x_{1} \eta_{a} x_{2} \ldots \eta_{a} x_{n-1} \eta_{a} x_{n}=x .
$$

We have two cases: 
Case 1. Set $x \in(H-\mathcal{C})$, so $(H-\mathcal{C}) \subseteq P(x)$. From (4.1) we have $z \eta_{a} x_{2}$ whence by Proposition 3.4, $x_{2} \in P(z)$. If we suppose that $z \in a^{n} \subseteq \mathcal{C}$, then $P(z)=a^{n}$ thus $x_{2} \in a^{n}$. Using again (4.1) we would obtain $x \in a^{n} \subseteq \mathcal{C}$ and it is a contradiction. Therefore $z \in(H-\mathcal{C})$ and hence $z \in P(x)$.

Case 2. Set $x \in a^{n} \subseteq \mathcal{C}$, so $P(x)=a^{n}$. By Proposition 3.4 and (4.1), we have $z \in a^{n}$ and hence $P(z)=a^{n}$. Therefore $z \in a^{n}=P(x)$.

Hence $\phi_{c}^{-1}\left(\phi_{c}(P(x))\right) \subseteq P(x)$ and since the converse inclusion holds obviously, it follows that $P(x)=\phi_{c}^{-1}\left(\phi_{c}(P(x))\right)$ and thus the proof is complete. $\square$

\section{REFERENCES}

1. Corsini, P. - Hypergroupes d'associativite' de quasigroupes mediaux, Convegno su Sistemi Binari e Loro Applicazioni, Taormina, 1978, 7-22.

2. Corsini, P. - Prolegomena of hypergroup theory, Supplement to Riv. Mat. Pura Appl. Aviani Editore, Tricesimo, 1993.

3. Corsini, P.; Leoreanu, V. - Applications of Hyperstructure Theory, Advances in Mathematics (Dordrecht), 5, Kluwer Academic Publishers, Dordrecht, 2003.

4. Corsini, P.; Romeo, G. - Hypergroupes completes et, $\mathfrak{F}$-groupoides, Convegno su Sistemi Binari e Loro Applicazioni, Taormina, 1978, 129-146.

5. Davvaz, B.; Karimian, M. - On the $\gamma_{n}^{*}$-complete hypergroups, European J. Combin., 28 (2007), 86-93.

6. Davvaz, B.; Karimian, M. - On the $\gamma_{n}$-complete hypergroups and $K_{H}$ hypergroups, Acta Math. Sin. (Engl. Ser.), 24 (2008), 1901-1908.

7. FRENI, D. - Une note sur le coeur d'un hypergroupe et sur la cloture transitive $\beta^{*}$ de $\beta$, [A note on the core of a hypergroup and the transitive closure $\beta^{*}$ of $\beta$, Riv. Mat. Pura Appl., 8 (1991), 153-156.

8. Freni, D. - A new characterization of the derived hypergroup via strongly regular equivalences, Comm. Algebra, 30 (2002), 3977-3989.

9. FRENI, D. - Strongly transitive geometric spaces: applications to hypergroups and semigroups theory, Comm. Algebra, 32 (2004), 969-988.

10. Koskas, M. - Groupes et hypergroupes homomorphes à un demi-hypergroupe, C.R. Acad. Sci. Paris, 257 (1963), 334-337.

11. Koskas, M. - Groupoides, demi-hypergroupes et hypergroupes, J. Math. Pures Appl., 49 (1970), 155-192

12. Karimian, M.; Davvaz, B. - On the $\gamma$-cyclic hypergroups, Comm. Algebra, 34 (2006), 4579-4589. 
13. LeOreanu, V. - Centralisateur d'un élément dans un hypergroupe réversible (French) [The centralizer of elements in reversible hypergroups], Rend. Circ. Mat. Palermo, 43 (1994), 413-418 (1995).

14. Leoreanu-Fotea, V.; DAvvaz, B. - n-hypergroups and binary relations, European J. Combin., 29 (2008), 1207-1218.

15. Migliorato, R. - Semi-ipergruppi e Ipergruppi n-completi, Ann. Sci. Univ. Clermont II, Sèr Math., 23 (1986), 99-123.

16. Marty, F. - Sur une Generalization de la Notion de Groupe, 8th Congress Math. Scandinaves, Stockholm, Sweden, 1934, 45-49.

17. Romeo, G. - Limite diretto di semi-ipergruppi e iperguppi di associativita', Riv. Math. Univ. di Parma, 8 (1982), 281-288.

18. Vougiouklis, T. - The fundamental relation in hypergroups. The general hyperfield, Proc, 4th Int. Congress Algebraic Hyperstructures and Applications, Xanthi, 1990, World Scientific, 209-217, 1991.

19. Vougiouklis, T. - Hyperstructures and Their Representations, Hadronic Press Monographs in Mathematics, Hadronic Press, Inc., Palm Harbor, FL, 1994.

20. Zhan, J.; Davvaz, B.; Shum, K.P. - A new view of fuzzy hypermodules, Acta Mathematica Sinica, English Series, 23 (2007), 1345-1356.

Received: 9.XI.2012

Revised: 23.IV.2013

Accepted: 29.IV.2013
Mathematics Department, Shahid Bahonar University of Kerman,

IRAN

smousavi@mail.uk.ac.ir

Faculty of Mathematics, "Al.I. Cuza" University of Iaşi,

Bd. Carol I, nr. 11, Iaşi, ROMANIA

foteavioleta@gmail.com

Mathematics Department, Vali-e-Asr University of Rafsanjan,

IRAN

m.j@mail.vru.ac.ir 